\title{
Polarimetry as a Diagnostic of Circumstellar Envelopes
}

\author{
Karen S. Bjorkman \\ Ritter Observatory, Dept. of Physics \& Astronomy, University of \\ Toledo, Toledo, $\mathrm{OH} 43606$, USA
}

\begin{abstract}
Polarimetric observations of stars can provide useful diagnostics of the geometry and physical conditions of the circumstellar envelope. This paper reviews the application of polarimetric techniques to hot stars (with particular emphasis on Be stars) and the status of polarization observations, and discusses some recent developments that improve our ability to make and to interpret such observations. Polarization measurements, bolstered by the increasing availability of new instruments on larger telescopes, continue to improve toward both higher signal-to-noise ratio and higher spectral resolution. Because of this we can begin to address specific questions of the physics of the circumstellar envelopes, since polarization provides a means to separate the effects of the photosphere from those of the envelope. New theoretical and analytic approaches are improving our ability to interpret spectropolarimetric data. We now are able to determine characteristics such as disk opening angle, density, and temperature. Furthermore, polarimetric observations in new wavelength regimes (e.g. ultraviolet and infrared) are opening new avenues to investigate circumstellar disks. The combination of spectropolarimetry with other kinds of data, such as spectroscopy, photometry, and interferometry, shows promise for providing stringent tests for theoretical models of the circumstellar envelopes of Be stars.
\end{abstract}

\section{Introduction}

When discussing the circumstellar envelopes (CSE) of hot stars, and especially $\mathrm{Be}$ stars, it is apparent that one of the fundamental problems we have in learning about them is the simple fact that in most cases the envelopes are unresolved by current techniques. While the ongoing developments in optical interferometry suggest that this will not be the case for much longer, at least for nearby stars, we still must find techniques that will allow us to investigate the nature of the envelopes without necessarily being able to resolve them directly.

Since the total light we observe consists of the combination of direct starlight plus any light scattered by the envelope into the line of sight, and since the process of scattering polarizes the non-direct contribution, we can use polarization observations as a means of separating out the effects of the envelope. Through the scattering and attenuation process, a signature of the envelope material and conditions becomes imprinted on the polarized light; thus, polarization data can provide insight into the composition, temperature, density, and physical geome- 
try of the circumstellar material, even when the CSE cannot be resolved directly through imaging. However, one complication is that the interstellar medium also polarizes starlight, and so we must be able to adequately remove the interstellar contribution in order to study the effects of the CSE alone.

\section{Polarization Diagnostics}

Polarigenic mechanisms, such as scattering, imprint a signature of circumstellar material on the light in several ways. First, the total number of scatterers, combined with their distribution in space, determines the overall level of polarization observed. Second, the characteristics of the scattering material, as well as of the illuminating source, will affect the wavelength-dependence of the polarization. These general principles can be applied to the interpretation of spectropolarimetric observations of hot stars.

\subsection{Effects of the Scattering Geometry}

The distribution of the scattering material around the star will affect the resulting polarization. For example, in the case of a uniform spherical distribution of scatterers, the net polarization will sum to zero, and the observed light will be unpolarized. If the scatterers are distributed in an optically thin equatorial disk, then the polarization position angle will be perpendicular to the orientation of the disk. In this case, the overall level of the polarization will depend on the amount of cancellation caused by contributions due to scatterers in the polar regions. In the case of "blobs" in the circumstellar envelope, the resulting polarization will depend on the details of the blob distribution.

\subsection{Wavelength Dependence}

As discussed by Nordsieck et al. (1992), there are four basic means by which the polarization can become wavelength-dependent: 1) dilution of the polarized light at particular wavelengths by the addition of unpolarized light from emission by surrounding material; 2) the presence of absorptive opacity that reduces the scattered light more than the direct starlight; 3) a changing geometry of illumination of the scatterers as a function of wavelength, such as would occur with starspots; or 4) a wavelength dependence of the scattering process itself, such as occurs for scattering by dust grains. In the case of hot stars, where the polarization mechanism is predominantly electron scattering, we usually need to consider only the first and second cases above.

Since electron scattering is a wavelength-independent process, it alone would not produce a wavelength-dependent polarization signature. However, the addition of unpolarized (unscattered) direct light from material outside the polarizing region will dilute the observed polarization, which is effectively just the ratio of the polarized (scattered) light to the total (direct + scattered) light. This dilution will result in a net decrease in polarization (depolarization) at all wavelengths where this additional direct light is present. For example, in $\mathrm{Be}$ stars the polarization is often observed to decrease across the $\mathrm{H} \alpha$ emission line. This is due to the diluting effect of $\mathrm{H} \alpha$ emission in the extended circumstellar envelope. 
The second effect resulting in wavelength-dependent polarization in hot stars is the presence of absorptive opacity. This can occur in two ways pre-scattering attenuation and post-scattering attenuation (Wood \& Bjorkman 1995). In the case of Be stars, the light which is scattered in the circumstellar disk must then escape out of the disk. As the escape path travels through additional disk material, the opacity of this disk material becomes imprinted on the scattered light spectrum. The dominant effect is hydrogen opacity, and this modifies the polarization spectrum into the characteristic "sawtooth" shape so clearly seen in spectropolarimetric observations, as predicted by Poeckert \& Marlborough (1979) and first observed in detail by Poeckert, Bastien, \& Landstreet (1979). However, hydrogen opacity is not the only contributor to this effect; the contributions of other opacity sources turn out to be a crucial diagnostic for the disk density and temperature, as will be discussed later.

\subsection{Time Variability}

The other useful polarization diagnostic for circumstellar envelopes is the time variability. Time-variable polarization provides clues to changes occurring within the envelope, and can place stringent constraints on proposed CSE models. It provides an accurate determination of the intrinsic position angle of the circumstellar envelope.

\section{Application to Hot Stars}

Many previous studies of the polarization of hot stars have laid the groundwork for our ability to interpret the new higher-quality polarization data available today. It would require far more than the space available here to discuss all of this work in detail, but interested readers are referred to discussions and references found in Gehrels (1974) and Coyne et al. (1988) for background. Here we present only a brief and incomplete sample of some previous results, for the purpose of illustrating the contributions of polarimetry to the investigation of hot stars.

\subsection{OB Supergiants}

Broadband polarimetric studies by Hayes $(1984,1985,1986)$ and Lyot polarimetric observations by Lupie \& Nordsieck (1987) showed that OB supergiants had intrinsic polarization, and that this polarization was often variable in time. Recent spectropolarimetric observations of $\zeta$ Pup showed evidence for a depolarization effect across the $\mathrm{H} \alpha$ line (Harries \& Howarth 1996). From a long-term monitoring campaign, detailed spectropolarimetric observations of P Cygni by Taylor et al. (1991) showed evidence for aperiodic, asymmetric, apparently random blob ejections in the circumstellar envelope, often over very short (day-today) timescales. Interestingly, the suggested clumpy nature of such a wind was later confirmed by direct coronographic images of the CSE of P Cyg (O'Connor, Meaburn, \& Bryce 1998) and by interferometry (Vakili et al. 1997). 


\subsection{Wolf-Rayet Stars}

Polarimetric studies of Wolf-Rayet (WR) stars have been carried out by several groups. Robert et al. (1990; and references therein), Schulte-Ladbeck (1995; and references therein), and Harries, Hillier, \& Howarth (1998) are examples of some of these studies, although this certainly is not a complete list. From these studies, the CSE of some WR stars have be shown to be clumpy, while some others (e.g. EZ CMa; Schulte-Ladbeck et al. 1990) show evidence for enhanced equatorial density, possibly similar to the wind-compressed zone (WCZ) model suggested by Ignace, Cassinelli, \& Bjorkman (1996).

\section{3. $\quad B[\mathbf{e}]$ Stars}

Polarimetric observations of $\mathrm{B}[\mathrm{e}]$ stars, which have evidence of dust around them, have provided some of the key evidence in support of the suggested bipolar geometry of these systems (e.g. Zickgraf \& Schulte-Ladbeck 1989; Magalhães 1992; Oudmaijer \& Drew 1999). For an example of new high-resolution line spectropolarimetry of $\mathrm{B}[\mathrm{e}]$ stars, see the paper by Oudmaijer, these proceedings.

\subsection{Herbig Ae/Be Stars}

Herbig $\mathrm{Ae} / \mathrm{Be}(\mathrm{HAeBe})$ stars have been the subject of many polarimetric studies. Early broadband work by Vrba, Schmidt, \& Hintzen (1979; also references therein) demonstrated that $\mathrm{HAeBe}$ stars showed intrinsic polarization. Combined broadband polarimetric and photometric studies showed that in some cases there is an anti-correlation between polarization levels and photometric magnitudes, so that the star shows the highest polarization levels when it appears the faintest (Grinin 1994). This has been interpreted as evidence for blockage of the central star light, possibly by blobs or planetesimals in the CSE. Similar results have been found by Yudin \& Evans (1998) and others. More recently, spectropolarimetry has shown evidence for dense equatorial disks in some of the HAeBe stars, both on the basis of $\mathrm{H} \alpha$ line polarization effects (Oudmaijer \& Drew 1999) and on the basis of polarization "flips" with wavelength (Bjorkman \& Schulte-Ladbeck 1994). Imaging polarimetry has also played an important role in studies of the CSE of HAeBe stars.

\subsection{Binary Systems}

Several interesting results on high-mass binary systems have been determined from polarimetric studies. For example, Roche et al. (1997) found evidence for disk loss in the Be/x-ray binary X Per (see also Roche et al., these proceedings). Hoffman, Nordsieck, \& Fox (1998) used spectropolarimetry to separate the contributions to the complex spectrum of $\beta$ Lyr; by doing so, they were able to connect particular spectral features to specific origins within the system (see also Hoffman, Nordsieck, \& Whitney, these proceedings).

\subsection{Magnetic Field Searches}

Polarization can be used to look for evidence of magnetic fields in hot stars. Barker et al. (1985) used polarization measurements to place upper limits of about 100 gauss on any global magnetic fields in Be stars. Ignace, Cassinelli, \& Nordsieck (1999) and Ignace, Nordsieck, \& Cassinelli (1997) suggested using 
spectropolarimetry to look for the Hanle effect in resonance lines, which might permit the detection of magnetic fields as low as 1-10 gauss. Results of magnetic field searches using circular polarization measurements are discussed in papers by Mathys and by Henrichs, both in these proceedings.

\section{Application to Be Stars}

Since the primary focus of this meeting is on the Be phenomenon, the remainder of this paper will discuss the polarimetric results on Be stars. Polarization studies of Be stars have a long history, and review papers from earlier conferences nicely summarize the early results. For a review of results prior to 1976, see Coyne (1976). For a review of results from then until about 1982, see Coyne \& McLean (1982).

As of 1982, much progress had already been made toward exploiting the capabilities of polarimetry. Comprehensive broadband polarimetric surveys had been completed (e.g. Poeckert, Bastien, \& Landstreet 1979; Hayes 1980; Poeckert \& Marlborough 1976; McLean \& Brown 1978). Narrow-band line-scan polarimetry across the $\mathrm{H} \alpha$ and $\mathrm{H} \beta$ lines had been done (Poeckert \& Marlborough 1977; McLean et al. 1979; McLean \& Clarke 1979). The first full optical range spectropolarimetry (of EW Lac and $\gamma$ Cas) had been carried out (Poeckert et al. 1979; Poeckert \& Marlborough 1978a). Good initial models had been developed for interpreting the data (Capps, Coyne, \& Dyck 1973; Poeckert \& Marlborough 1978b; McLean 1979).

Since that time, a number of new developments have rekindled interest in the possibilities for using polarimetry to investigate the disks of Be stars. More (and better) polarimeters, especially spectropolarimeters, are becoming available, and many of them are beginning to be available on larger telescopes. These instrumental developments have led to improved signal-to-noise observations, higher spectral resolution, and larger wavelength coverage (see Figure 1), all of which are crucial to providing the important details required for model constraints. In addition, monitoring efforts with dedicated telescopes are providing an excellent baseline of better time coverage for more stars (for example, see Bjorkman, Meade, \& Babler, these proceedings).

Polarization measurements have now been extended into other wavelength regimes, most notably the ultraviolet (UV) and infrared (IR). Detailed Monte Carlo codes have been developed to improve modeling capabilities and to extend these into the new wavelength regimes; in particular, these provide the capability to include far more realistic physics, such as the effects of line opacities within the disk, as well as more realistic geometries for the distribution of circumstellar material.

\section{Current Efforts}

\subsection{Envelope Characteristics}

The wavelength dependence of the continuum polarization across a wide spectral range can be used to constrain the geometry (opening angle) and density of the circumstellar disk. Wood, Bjorkman, \& Bjorkman (1997) have demonstrated 


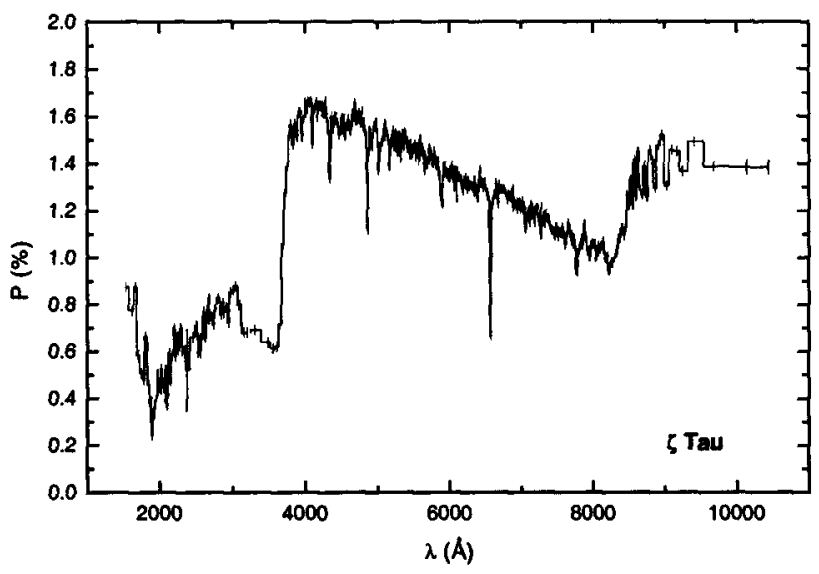

Figure 1. The polarization data for $\zeta$ Tau from the UV to the nearIR. This gives an example of the high signal-to-noise, moderate spectral resolution, wide wavelength coverage data that can be obtained with modern spectropolarimeters. Data have also been obtained at much higher spectral resolution, but with much less wavelength coverage. (Adapted from Wood et al. 1997)

this technique for the Be star $\zeta$ Tau. In this case, the spectropolarimetric continuum data (primarily in the optical) were modeled, together with the observed flux and IR photometry data from IRAS, in order to constrain acceptable models to two possibilities: a very thin disk (with an opening angle of about $3^{\circ}$ ), or a very thick disk (with an opening angle of about $57^{\circ}$ ). Combination of these limits with interferometric data from Quirrenbach et al. (1997) then showed that only the thin disk case could match all of the observational data.

\subsection{Analysis of UV Data}

The first UV spectropolarimetric observations of Be stars were obtained with the Wisconsin Ultraviolet Photo-Polarimeter Experiment (WUPPE) aboard the Astro-1 and Astro-2 space shuttle missions, as part of a science program including a wide variety of different types of stars. On Astro-1, 3 Be stars were observed in the UV, with surprising results. The UV polarization was found to be much lower than expected from hydrogen opacity models, with a strong depolarization region present (Bjorkman et al. 1991, 1993). This was attributed to the effects of metal line blanketing in the disk region. Since the 3 stars observed on Astro-1 were all of similar spectral type, it was important to broaden the distribution of spectral types and rotation velocities. This was accomplished with observations from Astro-2, which included $14 \mathrm{Oe} / \mathrm{Be}$ stars ranging in spectral type from 09.5 to $B 9$, and ranging in $v \sin i$ values between 50 and $400 \mathrm{~km} \mathrm{~s}^{-1}$.

The Astro-2 data clearly show a dependence of the UV depolarization on the spectral type (see Figure 2). This is consistent with the idea that the depolarization is due to the effects of line blanketing attenuation of the scattered light within the disk. Earlier spectral type stars show the largest line-blanketing effects in a region where Fe III is dominant (around 1600-1900 $\AA$ ), while for later 


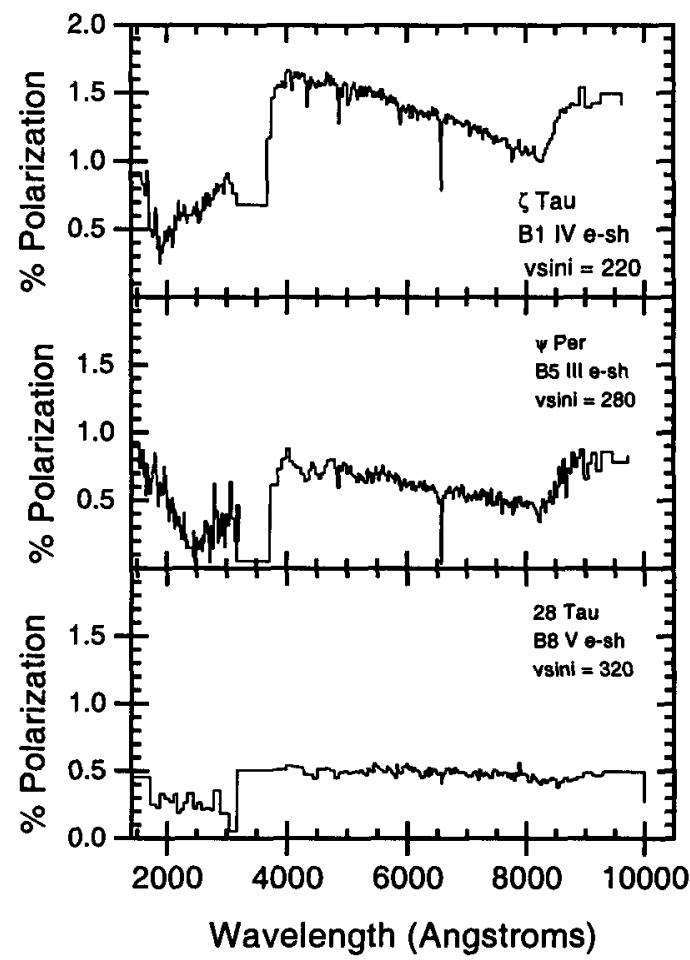

Figure 2. Examples of Be stars observed with WUPPE/Astro-2. Note the change in wavelength of the UV depolarization as a function of temperature.

spectral types, the line-blanketing depolarization shifts to Fe II regions (around $2600 \AA$ ). This is consistent with the similar photospheric line-blanketing effects discussed by Abbott (1982). As a result of this line-blanketing attenuation, the spectropolarimetric data provide an excellent probe of the disk material itself and can be exploited to try and determine characteristics such as average temperature and density within the disk. First attempts at interpreting these data are currently underway (Bjorkman, Bjorkman, \& Wood, these proceedings).

\subsection{Combination with Other Types of Observations}

The combination of spectropolarimetric data with other types of data has proven valuable in constraining models to unique results in ways that no single type of data alone can do. For example, interferometric results on Be stars have clearly resolved disks around several stars (e.g. Thom, Granes, \& Vakili 1986; Quirrenbach et al. 1993, 1994), but the vast majority of Be stars are still beyond the resolving power of these first generation instruments. While new instruments now starting to come on line will certainly improve the situation, polarimetry can provide a useful means of assessing the disk characteristics in the meanwhile. 

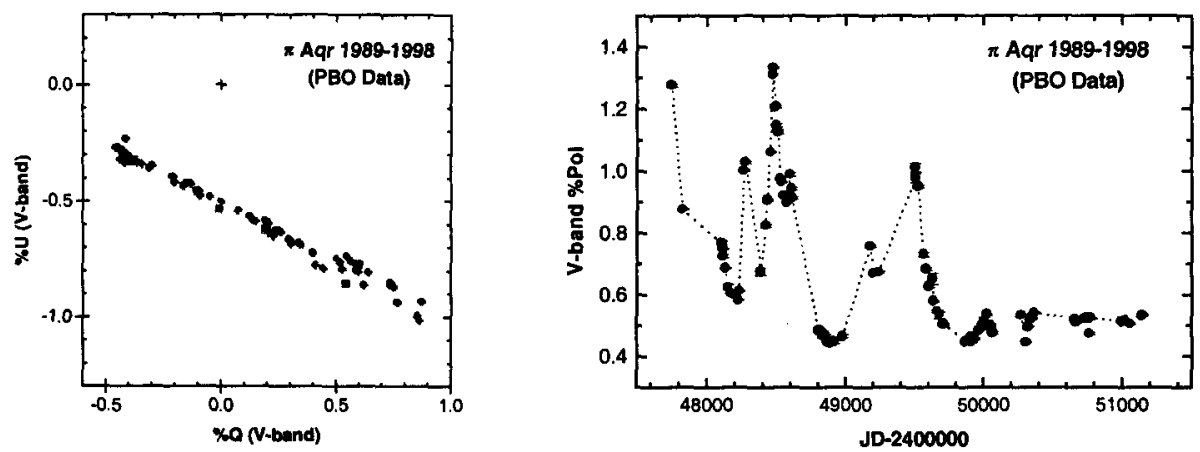

Figure 3. The polarimetric variability of $\pi$ Aqr from 1989-1998. a) The left panel shows the variations in the Stokes Q-U plane. The straight line defines the angle $2 \theta$, where $\theta$ is the position angle perpendicular to the disk position angle on the sky. b) The right panel shows the binned V-band equivalent polarization variations as a function of time. Note the relatively large polarimetric "outbursts" which occurred in 1989, 1991, and 1994. In recent years the star seems to have been in a polarimetrically quiescent state.

However, it is important to show that the polarization data give the same results as interferometry in cases where both can be carried out.

Quirrenbach et al. (1997) have demonstrated this by combining optical interferometry and optical spectropolarimetry for a set of seven Be stars. They were able to show conclusively that in at least these cases, the circumstellar envelopes of $\mathrm{Be}$ stars are definitely disks, not ellipsoids. Furthermore, their results demonstrate observationally that the polarization data alone can give the necessary information about the intrinsic position angle of the disk on the sky, as had been suggested previously on the basis of theory. This information is very helpful in planning for future interferometric or coronographic imaging programs.

\subsection{Time Variability}

Another aspect of the polarization data which can provide useful information is the time variability. In the case of $\pi$ Aqr, time variations in both the polarization (see Figure 3) and spectral data, along with changes in the optical and IR photometry, may signal the loss of the circumstellar disk (Bjorkman 1994; Bjorkman, Miroshnichenko et al., these proc.). Other stars also show dramatic variability in the polarization. However, to use such data effectively, long timescale monitoring of polarimetric properties of a large sample of Be stars must be undertaken. McDavid (1990, 1994, 1999) has provided useful long-term broadband polarimetric monitoring of Be stars. The spectropolarimetric monitoring program at the Pine Bluff Observatory (PBO) also has amassed a large database of variability data (Nordsieck et al. 1992; Bjorkman et al., these proceedings). Other monitoring programs must also be encouraged, and it is important to make these data generally available to the community for analysis. 
Models for circumstellar envelopes of Be stars, such as one-armed density waves, have been suggested to account for the well-known $V / R$ variations. However, these models also would predict polarimetric variability, and these predictions can be tested with observations (McDavid et al., these proceedings).

\section{Future Directions}

As future instrumentation improves our ability to make detailed polarimetric observations, and as our models get better, there are a number of areas where significant improvements in our understanding of circumstellar envelopes can be made. These areas have already been alluded to above, but for convenience they are summarized here:

1. Physical properties of the circumstellar disk, including the global distribution of material, the opening angle and thickness of the disk, and the average temperature, density, and composition of the material in the disk can be determined.

2. The disk orientation on the sky can be determined. This is very useful to know beforehand when planning coronographic or interferometric observations, which will become more and more important in the near future.

3. Estimates of inclination angles of the disks may be obtainable from combining polarimetry with photometric data.

4. Changes in the disk (or even loss of the disk) can be investigated,

5. It must be remembered that any models developed to explain spectroscopic (or other) variability also will have polarimetric consequences, and the predicted polarization behavior resulting from such models should be considered. Carried out in this manner. polarization observations can provide strong constraints for such models.

However, some concerns should be noted about future directions. It will be imperative to maintain telescope access for studies of relatively bright stars, a prospect that is becoming more and more bleak as many smaller telescopes are closed down in order to support the new generation of very large telescopes. Monitoring capability is important, and this can often only be provided by dedicated instruments and telescopes. Greater access to new instrumentation is necessary, and access to large telescopes for purposes of obtaining very high resolution, high signal-to-noise observations of bright stars must be pursued. Finally, strong support from theorists will be crucial if we are to fully exploit the potential of new and better polarization observations. Polarimetry can be difficult to interpret, and good, well-developed, readily available models will be extremely important in this endeavor.

Acknowledgments. I thank my collaborators, in particular Jon Bjorkman, Kenneth Wood, Marilyn Meade, Brian Babler, Ken Nordsieck, Andreas Quirrenbach, David McDavid, and Barbara Whitney, for many useful discussions on these topics over the years. Aspects of this work have been supported under NASA contract NAS5-26777 with the University of Wisconsin, and by NASA 
grants NAG5-3248 and NAG5-8054 to the University of Toledo. K.S. Bjorkman is a Cottrell Scholar of the Research Corporation, and gratefully acknowledges their support.

\section{References}

Abbott, D.C. 1982, ApJ, 259, 282

Barker, P.K., Landstreet, J.D., Marlborough, J.M., and Thompson, I.B. 1985, ApJ, 288, 741

Bjorkman, K.S. 1994, Ap\&SS, 221, 335.

Bjorkman, K.S., \& Schulte-Ladbeck, R.E. 1994, in ASP Conf. Ser. Vol. 62, The Nature and Evolutionary Status of Herbig Ae/Be Stars, ed. P.S. Thé, M.R. Pérez, \& E.P.J. van den Heuvel (San Francisco: ASP), 74

Bjorkman, K.S. et al. 1991, ApJ, 383, L67

Bjorkman, K.S. et al. 1993, ApJ, 412, 810

Capps, R.W., Coyne, G.V., \& Dyck, H.M. 1973, ApJ, 184, 173

Coyne, G.V. 1976, in IAU Symp. 70, Be and Shell Stars, ed. A. Slettebak (Dordrecht: Reidel), p. 233

Coyne, G.V., et al. 1988, Polarized Radiation of Circumstellar Origin (Vatican: Vatican Observatory)

Coyne, G.V., \& McLean, I.S. 1982, in IAU Symp. 98, Be Stars, ed. M. Jaschek \& H.-G. Groth (Dordrecht: Reidel), p. 77

Gehrels, T. 1974, Planets, Stars, and Nebulae Studied with Photopolarimetry (Tucson: University of Arizona Press)

Grinin, V.I. 1994, in ASP Conf. Ser. Vol. 62, The Nature and Evolutionary Status of Herbig Ae/Be Stars, ed. P.S. Thé, M.R. Pérez, \& E.P.J. van den Heuvel (San Francisco: ASP), 63

Harries, T.J., Hillier, D.J., and Howarth, I.D., 1998, MNRAS, 296, 1072

Harries, T.J., \& Howarth, I.D. 1996, A\&A, 310, 533

Hayes, D.P. 1980, PASP, 92, 661

Hayes, D.P. 1984, AJ, 89, 1219

Hayes, D.P. 1985, ApJ, 289, 726

Hayes, D.P. 1986, ApJ, 302, 403

Hoffman, J.L., Nordsieck, K.H., \& Fox, G.K. 1998, AJ, 115, 1576

Ignace, R., Cassinelli, J.P., \& Bjorkman, J.E. 1996, ApJ, 459, 671

Ignace, R., Cassinelli, J.P., \& Nordsieck, K.H. 1999, ApJ, 520, 335

Ignace, R., Nordsieck, K.H., \& Cassinelli, J.P. 1997, ApJ, 486, 550

Lupie, O.L., \& Nordsieck, K.H. 1987, AJ, 93, 214

Magalhães, A.M. 1992, ApJ, 398, 286

McLean, I.S. 1979, MNRAS, 186, 265

McLean, I.S. \& Brown, J.C. 1978, A\&A, 69, 291

McLean, I.S. \& Clarke, D. 1979, MNRAS, 186, 245

McLean, I.S. et al. 1979, ApJ, 228, 802 
McDavid, D. 1990, PASP, 102, 773

McDavid, D. 1994, PASP, 106, 949

McDavid, D. 1999, PASP, 111, 494

Nordsieck, K.H. et al. 1992, in ASP Conf. Ser. Vol. 22, Non-isotropic and Variable Outflows from Stars, ed. L. Drissen, C. Leitherer, \& A. Nota (San Francisco: ASP), 114

O'Connor, J.A., Meaburn, J., \& Bryce, M. 1998, MNRAS, 300, 411

Oudmaijer, R.D. \& Drew, J.E. 1999, MNRAS, 305, 166

Poeckert, R., Bastien, P., \& Landstreet, J.D. 1979, AJ, 84, 812

Poeckert, R., \& Marlborough, J.M. 1976, ApJ, 206, 182

Poeckert, R., \& Marlborough, J.M. 1977, ApJ, 218, 220

Poeckert, R., \& Marlborough, J.M. 1978a, ApJ, 220, 940

Poeckert, R., \& Marlborough, J.M. 1978b, ApJS, 38, 229

Poeckert, R., \& Marlborough, J.M. 1979, ApJ, 233, 259

Quirrenbach, A. et al. 1993, ApJ, 416, L25

Quirrenbach, A. et al. 1994, A\&A, 283, L13

Quirrenbach, A. et al. 1997, ApJ, 479, 477

Robert, C. et al. 1990, ApJ, 359, 211

Roche P. et al. 1997, A\&A, 322, 139

Schulte-Ladbeck, R.E. 1995, in IAU Symp. 163, Wolf-Rayet Stars: Binaries, Colliding Winds, Evolution, ed. K.A. van der Hucht and P.M. Williams (Dordrecht: Kluwer), p. 176

Schulte-Ladbeck et al. 1990, ApJ, 365, L19

Taylor, M. et al. 1991, AJ, 102, 1197

Thom, C., Granes, P., \& Vakili, F. 1986, A\&A, 165, L13

Vakili, F. et al. 1997, A\&A, 323, 183

Vrba, F.J., Schmidt, G.D., \& Hintzen, P.M. 1979, ApJ, 227, 185

Wood, K., \& Bjorkman, J.E. 1995, ApJ, 443, 348

Wood, K., Bjorkman, K.S., \& Bjorkman, J.E. 1997, ApJ, 477, 926

Yudin, R.V., \& Evans, A. 1998, A\&AS, 131, 401

Zickgraf, F.-J., \& Schulte-Ladbeck, R.E. 1989, A\&A, 214, 274

\section{Discussion}

P. Harmanec: If I am right with the concept of the pseudo-photosphere as the optically thick part of the varying envelope - would you be able to distinguish between the properties of the pseudo-photosphere and the properties of the optically thin outer parts of the envelope from your polarimetric observations and analysis?

K. Bjorkman: The polarization that we observe is produced mostly in the very inner regions (within a few stellar radii) of the envelope. If the star were to develop an extended oblate pseudo-photosphere, as you suggest, there would certainly be polarimetric consequences. Electron scattering atmospheres can pro- 
duce polarizations of up to $11 \%$ at the limb. Normally, an integrated polarization is zero because the star is spherical. But for an oblate pseudo-photosphere, the cancellation would not be complete and a net polarization would result. Depending on the oblateness, this could produce polarizations of maybe $0.1-1 \%$ or so, which are measurable. So I would say that it certainly ought to be possible to study such effects with polarimetry. To try and distinguish between polarization changes from an oblate pseudo-photosphere and changes in a circumstellar disk, you would have to take into account other types of observational information as well, such as changes in spectroscopic line profiles or IR excesses.

L. Balona: To what extent does the assumption of a simple geometry, parameterized by the opening angle, affect your solution of the physical parameters of the disk?

K. Bjorkman: It is true that if we assumed a different basic geometry, we might get quantitatively different results for the density and thickness (geometrical opening angle) of the disk, but the basic requirement of a thin disk (or equivalent geometry) will remain if we are to adequately fit the other observational data.

R. Ignace: (in response to Balona's comment) Models often assume a constant disk opening angle with radius, and it is true that polarization is only sensitive to that angle at the inner disk. But having determined this angle there, its constancy can be tested by comparing predicted IR excess with observations.

J. Zorec: I wonder whether the geometrical constraints you have derived for circumstellar envelopes in Be stars by fitting spectropolarimetric observations are strongly model dependent. Instead of a disk with an opening angle, you may have an ellipsoidal-like structure which also produces bi-valued polarization as a function of aspect angle. I do not know if there are criteria clear enough to distinguish from polarimetric data between them both.

K. Bjorkman: The exact numbers for the disk opening angle are probably model-dependent. However, the qualitative requirement for a thin disk is not. It is true that if all you look at is one type of observation, whether it be polarimetric, spectroscopic, or anything else, you will probably be able to find a model to fit it. But if you require that model to simultaneously fit other types of data as well, it is much harder to do. What we have tried to do is to narrow down the range of models that are acceptable by trying to simultaneously fit several different types of observational data. We have looked at ellipsoidal models (and by that, I mean only slightly flattened envelopes), and they clearly cannot explain the observations. First, I refer you to our combined interferometry and spectropolarimetry paper (Quirrenbach et al. 1997), where you will find that ellipsoidal models were ruled out by the interferometry directly. Second, in our models of $\zeta$ Tau, we found that "thick disk" models, with opening angles of $50^{\circ}$ or more, were unable to reproduce the IR excesses very well. The only models that did work were thin disks, with opening angles of about $3^{\circ}$. Of course, an ellipsoid that is extremely flattened, having an "effective opening angle" of only $3^{\circ}$ would also work, but that would be geometrically the same as a thin disk anyway. 